\title{
La construction du rapport de genre aux loisirs sportifs et à la santé dans une population scolarisée de Guadeloupe
}

The Construction of Gender Identity in Recreational Sports and Health in the Schooled Population of Guadeloupe

Sylvain Ferez, Sébastien Ruffié et Michel Le Her

\section{OpenEdition Journals}

Édition électronique

URL : http://journals.openedition.org/rref/823

DOI : $10.4000 /$ rref.823

ISSN : $2494-7830$

Éditeur

CRDP de Guadeloupe

Édition imprimée

Date de publication : 15 décembre 2009

Pagination : 13-25

ISSN : 1962-2864

Référence électronique

Sylvain Ferez, Sébastien Ruffié et Michel Le Her, « La construction du rapport de genre aux loisirs sportifs et à la santé dans une population scolarisée de Guadeloupe ", Recherches et ressources en éducation et formation [En ligne], 3 | 2009, mis en ligne le 24 avril 2020, consulté le 15 octobre 2020 URL : http://journals.openedition.org/rref/823 ; DOI : https://doi.org/10.4000/rref.823

\section{(2) $(1) \Theta$}

La revue Recherches et ressources en éducation et formation est mise à disposition selon les termes de la Licence Creative Commons Attribution - Pas d'Utilisation Commerciale. 


\title{
LA CONSTRUCTION DU RAPPORT DE GENRE AUX
} LOISIRS SPORTIFS ET A LA SANTÉ DANS UNE POPULATION SCOLARISÉE DE GUADELOUPE

\author{
S. FEREZ (1), S. RUFFIÉ (2) ET M. LE HER (2)
}

(1) Sant.E.Sih., JE 2516, Université de Montpellier 1, 700 avenue du Pic Saint-Loup, 34090 Montpellier

(2) Laboratoire ACTES, UPRES EA 3596, Université Antilles-Guyane, Campus de Fouillole, BP 250, 97157 Pointe-a-Pitre Cedex

Contact : sylvain.ferez@univ-montp1.fr

Résumé : Cette recherche a été réalisée auprès d'une population de jeunes Guadeloupéens scolarisés. Elle vise à évaluer le poids de l'identité de genre dans les comportements sanitaires et plus particulièrement dans des pratiques physiques et sportives. Une enquête, à partir d'un questionnaire élaboré spécifiquement pour les besoins du travail, a été proposée à des collégiens et lycéens $(\mathrm{n}=454)$. Une analyse des tris croisés a été effectuée à partir du logiciel Modalisa, mettant en évidence les liens significatifs. L'ensemble des résultats montre qu'outre sur l'engagement sportif, la construction des identités de genre a des effets importants sur les modes de sociabilité et les rapports aux pratiques culturelles et de loisir. Elle ne semble par contre pas influencer directement les habitudes alimentaires et sanitaires. Au final, cette étude montre comment l'espace sportif offre avant tout un lieu d'incorporation de la structure sociale de genre, dans laquelle les réseaux amicaux et la socialisation secondaire prennent le relais de la socialisation primaire du genre au sein de la famille.

Mots clefs : Identité de genre, sport, Guadeloupe.

\begin{abstract}
This research has been achieved among a population of young Guadeloupean school pupils. It aims to evaluate the importance of gender identity in their sanitary behaviour and especially in their physical and sport practices. A questionnaire adapted to the subject of the study has been submitted to secondary school pupils $(\mathrm{n}=454)$. An analysis of crossed stortings, putting the emphasis on the significant links, has been obtained thanks to Modalisa software. As a whole, the results of the questionnaire show that, the construction of gender identité, among Guadeloupean schoolchildren, has major effects not only on commitent to sport practices but also on sociability and the relationship to cultural practices. Yet, it doesn't seem to have direct impact on nutritional and sanitary habits. The present study shows how the sport space primarily offers an opportunity for integrating social gender structure. At last, the study shows how the sport space offers especially a situation for incorporation of the social gender structure. In such structures, friendly relationship between friends and secondary socialization relay gender Relationship within the family.
\end{abstract}

Key words : Gender identity, sport, French West Indies (Guadeloupe). 


\section{INTRODUCTION}

Il existe peu de données sur le surpoids et l'obésité en Guadeloupe. L'ensemble de ces données sont regroupées dans le dernier Plan Régional de Santé Publique de la Guadeloupe (2004-2008, 42-43) dans un chapitre intitulé " alimentation et nutrition ». En 2000, seule la prévalence de l'obésité dans une population adulte de l'île était évaluée (sur une population de personnes de 18 à 76 ans fréquentant un centre de santé), et concernait 19\% de l'échantillon étudié. Entre novembre 2001 et novembre 2003, l'étude « Prévalence, prise en charge de l'hypertension artérielle et précarité ", réalisée auprès d'adultes, y évalue en moyenne la surcharge pondérale à $41 \%$ chez les hommes et à $58 \%$ chez les femmes. L'obésité est pour sa part (IMC $>30)$ située à $12 \%$ chez les hommes et $29 \%$ chez les femmes. Comment expliquer une surcharge pondérale plus importante des femmes que des hommes en Guadeloupe ? L'enquête effectuée par le service de la santé scolaire et l'ODESSA en 20002001 révélait pour sa part un surpoids important chez les enfants de 11 ans et de 15 ans. Aucune étude ne s'est cependant intéressée aux habitudes sanitaires et à «l'hygiène de vie » des jeunes Guadeloupéens.

Parallèlement, dans un contexte local marqué par l'importance des maladies cardio-vasculaires, la problématique du surpoids ne saurait être traitée sans prendre en considération, outre sa relation avec les aspects alimentaires, son lien avec l'activité physique ou la sédentarité. L'enjeu est finalement ici d'évaluer ce que les habitudes alimentaires, les pratiques sanitaires et les loisirs physiques des jeunes Guadeloupéens doivent à leur socialisation de genre. Une première hypothèse est que le surpoids et l'obésité sont en partie l'effet de styles de vie largement orientés par la construction des identités de genre via les interactions scolaires et extra-scolaires dans les groupes de pairs. Une seconde hypothèse est que le sport joue un rôle prépondérant dans la construction de ces styles de vie liés au genre.

\subsection{Activité physique, surpoids/obésité et styles de vie}

Nombreuses sont les études qui s'intéressent aux relations entre activité physique, attitudes sanitaires et styles de vie. Les données scientifiques sur l'obésité et le surpoids chez les enfants et adolescents s'intéressent à la fois aux déterminants et aux effets de ces derniers. Du côté des effets, les auteurs décrivent d'une part leurs conséquences psychologiques - en termes d'estime de soi, de bien-être, de qualité de vie, bref de « santé psychologique »- et d'autre part leurs conséquences sociologiques - relatant des processus d'exclusion sociale et de stigmatisation qui leur sont associés (Ball et al., 2004 ; Beech et al., 2004 ; Gordon-Larsen, 2001 ; Perry et al., 2004 ; Strauss, 2000 ; Strauss et al., 2003).

Du côté des déterminants, les études soulignent le poids de constructions socioculturelles qui pèsent aussi bien au niveau des habitudes que des perceptions. Ces constructions sont reliées à des indicateurs liés à l'origine ethnique, géographique, socioéconomique et à la socialisation de genre (Ara et al., 2007 ; Ball et al., 2004 ; Beech et al., 2004 ; Dowda et al., 2001 ; Gordon-Larsen, 2001 ; Padgett \& Biro, 2001 ; Paeratakul et al., 2005 ; Sanchez-Johnsen et al., 2004 ; Simeon et al., 2003). Plus globalement, l'impact de l'activité physique et/ou de la sédentarité semble en rapport avec ces indicateurs et la manière dont leur articulation se traduit en termes de styles de vie.

Ainsi, si l'activité physique ou la sédentarité peuvent être étudiées en eux-mêmes (pour mesurer leurs liens directs avec le surpoids et l'obésité), en montrant par exemple que la participation sportive scolaire ou extrascolaire réduit le risque de surpoids chez un jeune public (Bonaccorsi et al. ; 2007 ; Dowda et al., 2001 ; Taylor et al., 2006) ou qu'il existe une corrélation entre capacité aréobie et IMC (Ara et al., 2007), il paraît préférable de repérer leur inscription dans des modes de vie les associant à d'autres comportements et attitudes.

La plupart des études observent un impact du genre sur la relation entre surpoids/obésité et activité/inactivité physique. Gordon-Larsen (2001) montre que des filles obèses passent moins de temps en activité physique modérée et sont plus inactives que celles qui ne le sont pas. Il pointe par ailleurs les effets combinés du genre et de l'ethnicité sur la relation entre alimentation, surpoids/obésité et activité/inactivité physique : les adolescentes américaines d'origine caucasienne de son échantillon ont ainsi une dépense calorique hebdomadaire supérieure à celles des latino-américaines, tandis qu'elles présentent des niveaux d'activité physique plus élevés que les afro-américaines. Ara et al. (2007) ne trouvent, de leur côté, aucune différence significative entre garçons actifs et sédentaires, mais mesurent par contre une moindre obésité chez les filles actives que chez les sédentaires. L'activité physique ne semble cependant pas ici la variable qui influence le plus l'IMC, puisque qu'elle n'a un impact que sur celui des garçons. La capacité aérobie (VO2 max) est par contre corrélée à l'adiposité chez les deux sexes.

Dans l'étude de Kosti et al. (2007), le nombre de repas journaliers est associé au surpoids et à l'obésité pour les garçons, alors que la fréquence de prise du petit déjeuner, la fréquence de consommation de volaille et le temps passé dans des activités excluant le sport le sont pour les filles. Dowda et al. (2001) trouvent un lien significatif entre augmentation du temps passé devant la télévision et surpoids seulement chez les filles. D'autres notent enfin que si les niveaux d'activité physique des enfants déclinent avec l'âge, ce déclin est plus prononcé chez les filles. En vieillissant, l'écart de genre en matière d'investissement sportif tend donc à s'accroître, et les filles s'engagent moins volontiers dans des activités physiques ou des programmes d'entraînement favorisant la dépense énergétique (Perry et al., 2004). 


\section{S. FEREZ, S. RUFFIÉ ET M. LE HER RECHERCHES}

\subsection{Sport, masculinité et construction du genre}

Dans cet article, l'enjeu est d'évaluer l'importance de cette surdétermination de genre dans le rapport à la pratique sportive pour tenter de saisir certaines logiques sanitaires. Autrement dit, il s'agit de tenter de voir dans quelle mesure le rapport à l'activité physique s'inscrit, chez les jeunes Guadeloupéens et Guadeloupéennes, dans la construction (ou non) d'un profil sanitaire qui est en rapport (ou non) avec l'expression d'une identité de genre inscrite dans des rapports sociaux sexués. L'histoire et la sociologie du sport révèle combien ce domaine d'activités prend sens (en-deçà de toute signification sanitaire) au regard de la construction d'identités de genre entérinant les rapports sociaux de sexe. Les sports se construisent ainsi historiquement comme une citadelle masculine, et restent, malgré les évolutions au cours du $20^{\text {ème }}$ siècle, associés à l'expression d'une masculinité fondée sur un postulat hétérosexiste (Ferez, 2007, 2008 ; Terret, 2005). Les politiques pour l'équité de genre se heurtent ici fréquemment à une réalité qui résiste (Larson, 2006). En dépit de la mise en avant de l'équité comme valeur partagée, des inéquités persistent dans la culture des organisations sportives (Hoeber, 2007).

Robertson (2003) pointe notamment le rapport entre la socialisation masculine au travers du sport et les attitudes misogynes et homophobes. Une enquête américaine basée sur l'auto-déclaration d'actes d'agression et de coercition sexuelle révèle ainsi par exemple que l'investissement des garçons dans des sports agressifs au lycée est corrélé avec un plus grand recours à la violence psychologique et à la violence physique, avec le fait d'être à l'origine de davantage de coups et de blessures, mais aussi avec des attitudes plus sexistes, une plus forte hostilité à l'égard des femmes (avec une adhésion plus forte aux mythes attribuant la responsabilité du viol aux attitudes féminines) et à une moindre tolérance à l'égard de l'homosexualité (Forbes et al., 2006).

L'éducation physique scolaire, essentiellement centrée sur le sport, participe elle-même à la reproduction de la structure de genre (Ferez, 2004). Elle s'inscrit dans une division du travail et des orientations scolaires fortement sexuées. Des travaux constatent pour leur part une relation entre l'engagement en sport et le succès scolaire dans le domaine scientifique chez les lycéennes. La pratique du sport est dès lors envisagée comme une préparation à la culture masculine dans les classes de sciences (Hanson, 2007). Il apparaît simultanément que les carrières et le leadership des enseignants d'éducation physique contribuent à la fois à faire évoluer et à reproduire la perception dualiste du genre (Webb \& Macdonald, 2007). Plus globalement, les terrains sportifs demeurent des lieux de reconnaissance des comportements de genre " appropriés » et favorisent les stratégies d'hyper-ritualisation incitant à coller aux stéréotypes sexués. Chez les enfants, l'accès à ces derniers laisse d'ailleurs plus de latitude aux filles qu'aux garçons, qui doivent se conformer à ce qu'on attend d'eux (Schmalz \& Kerstetter, 2006). Les attentes paternelles apparaissent en outre comme un facteur déterminent dans le succès des carrières sportives (Coakley, 2006).

Ici, les écarts de performances entre hommes et femmes ne suffisent ni à expliquer, ni à justifier la ségrégation des sexes dans les épreuves. Les mesures de vérification du genre dans le sport de haut niveau attestent au contraire du fait que la construction de genre est un trait constitutif du sport compétitif (Müller, 2006). Cette construction a par ailleurs des effets à la fois sur l'accès des femmes au sport et sur la signification des disciplines qu'elles investissent. Liston (2006) montre ainsi, en Irlande, l'existence d'une hiérarchie entre disciplines sportives qui dépend de la position occupée par les athlètes féminines et des répercussions sociales de cette position en termes de perception masculine ou féminine de la discipline.

D’une façon générale, le caractère sexué des activités dépend de leur histoire et des contextes sociaux où elles s'insèrent. En Angleterre, la pratique du football est emblématique de la masculinité (Clark \& Paechter, 2007 ; Williams, 2006). Aux Etats-Unis, c'est le football américain qui, en contexte scolaire, figure le principal espace de construction de la subculture masculine (Clayton \& Humberstone, 2007), alors que le cheerleading (« pompom girl ») est, en dépit de sa sportivisation, associé à la féminité (Grindstaff \& West, 2006). Au Canada, la pratique masculine de la remise en forme pour élargir et sculpter le corps, souvent associée à une pratique de supplémentation comportant des risques au plan sanitaire, est décrite comme une réponse à la crise de la masculinité dans les classes moyennes (Atkinson, 2007).

Pour l'heure, aucune étude n'a été faite sur la signification de genre des activités physiques et sportives en Guadeloupe. Les travaux existant attestent cependant de l'image très positive de ces dernières sur l'île. Le sport y apparait à la fois un moyen de réussite et, en contexte post-colonial, comme un puissant vecteur de l'identité locale, comme en atteste la fierté liée à la mise en avant des nombreux champions issus de la région (Dumont, 2002). Dans ce cadre, une étude sur l'accès au sport de populations handicapées physiques montre comment il est utilisé en vue de construire une visibilité active qui semble exclure les femmes (Ruffié et al., soumis). Des travaux d'anthropologie culturelle sur le corps, la sexualité et la maladie soulignent par ailleurs le poids des rôles sexués traditionnels et de la stigmatisation de l'homosexualité aux Antilles (Giraud, 1999; Mulot, 2000 ; Pourette, 2002, 2004). Dans quelle mesure la pratique sportive participe-t-elle, ou non, à ces constructions culturelles ? Plus globalement, la socialisation de genre oriente-elle le rapport aux loisirs, à la sociabilité, aux modes de vie des collégiens et lycéens en Guadeloupe ? La variable sexuée a-t-elle un impact sur les habitudes alimentaires, les formes de sociabilité et les pratiques sanitaires et culturelles des jeunes Guadeloupéens? 


\section{MÉTHODE}

Pour répondre à ces questions, une enquête a été réalisée auprès d'une population de collégiens et lycéens de plusieurs établissements de la Grande Terre. Elle concerne 454 sujets, avec une proportion quasiment équivalente de garçons $(\mathrm{n}=229)$ et de filles $(\mathrm{n}=225)$. L'étude concerne des élèves nés entre 1996 et 1988 (11-20 ans) qui se trouvent en classe de $6^{\text {ème }}(3.1 \%)$, de $5^{\text {ème }}(31.1 \%)$, de $4^{\text {ème }}(25.3 \%)$, de $3^{\text {ème }}(28,2 \%)$, de $2^{\text {nde }}(0.9 \%)$, de $1^{\text {ère }}$ $(7.5 \%)$ et de Terminale (4.0\%). Elle se centre sur des variables socioculturelles afin d'évaluer des attitudes et comportements qui peuvent être impliqués dans la prévalence de l'obésité et du diabète. Les données ont été recueillies à partir d'un questionnaire s'intéressant aux comportements, aux savoirs et aux attitudes des enquêtés, et plus spécifiquement à leur rapport à la sédentarité et à l'activité physique. Ce questionnaire a été élaboré pour les besoins spécifiques de l'enquête, et administré par les chercheurs lors de créneaux d'une heure avec quelques étudiants ou avec des groupes classes d'une vingtaine de jeunes. Les 111 questions posées concernaient cinq grands indicateurs :

\subsection{Statut socioéconomique, éducation et position dans la fratrie}

Outre la mention du statut socioéconomique des parents, des informations ont été collectées sur le rang de naissance, le niveau d'étude des frères/sœurs et les aspirations professionnelles du répondant.

\subsection{Niveau d'activité physique ou de sédentarité}

Plusieurs questions visaient à évaluer l'activité physique effective, le type d'activités régulièrement pratiquées en dehors de l'école, le temps dédié à ces dernières, mais aussi les lieux de pratique et les partenaires impliqués. D'autres visaient davantage à saisir les goûts et les motivations pour la pratique. Une question concernait la fréquence hebdomadaire d'élévation significative du rythme cardiaque. Une autre interrogeait sur le moyen d'aller à l'école et, pour ceux s'y rendant à pied, sur le temps du trajet. Le temps d'inactivité était, de son côté, évalué à partir de questions portant sur le temps passé à regarder la télévision, à jouer à des jeux vidéos ou à utiliser Internet.

\subsection{Habitudes culturelles}

Le questionnaire s'intéressait par ailleurs à différents aspects liés à la culture et à l'origine ethnique. Le type de nourriture le plus fréquemment consommé, ainsi que celui préféré par le sujet, était demandé, tout comme les langues couramment maîtrisées et utilisées en contexte scolaire, amical et familial. Plusieurs questions permettaient enfin de mesurer à la fois les affiliations et les pratiques religieuses.

\subsection{Réseaux amicaux, pratiques de loisirs et usages culturels}

Un certain nombre d'informations collectées était en rapport avec les réseaux amicaux. Il s'agissait à la fois de connaître leur taille (avec deux questions sur le nombre de " très bons amis » garçons et filles), mais également leur provenance (l'école, le voisinage, la pratique associative, la communauté religieuse, etc. ?) et leurs origines ethniques et/ou religieuses. Une variable s'intéressait enfin aux types d'activités partagées avec eux.

\subsection{Santé et alimentation}

Concernant la santé, les personnes interrogées devaient préciser les différents types de médecines utilisés et de thérapeutes fréquentés, et avec qui ils s'y rendaient. Les habitudes alimentaires étaient également questionnées en s'intéressant à la prise du petit-déjeuner, au nombre de repas journalier, ainsi qu'au temps et la posture de prise de ces derniers (assise ou debout). Les sujets étaient invités à notifier les boissons consommées, en précisant la quantité ingérée chaque jour. Une question portait enfin sur la consommation d'alcool et plusieurs sur les rythmes de vie (heure de lever et de coucher en période scolaire et le week-end ou en période de vacances).

Pour répondre aux questions posées sur les liens existant entre le genre, les pratiques et habitudes sanitaires, les styles de vie et l'engagement dans les loisirs sportifs, nous proposons ici de traiter l'ensemble des tris croisés avec la variable « sexe du répondant ». Seuls ceux qui sont significatifs $(\mathrm{p}<.05)$ au test du Khi2 seront présentés. Un certain nombre de résultats ont été conservés, en dépit d'effectifs théoriques insuffisants pour attester d'effets significatifs, parce qu'ils présentaient des tendances pertinentes au regard du système d'interprétation élaboré. L'ensemble des traitements statistiques a été réalisé à partir du logiciel d'analyse de données Modalisa. 


\section{S. FEREZ, S. RUFFIÉ ET M. LE HER}

\section{RÉSULTATS}

Il est remarquable de constater que, sur l'ensemble des questions posées, une seule variable liée à l'alimentation différencie les garçons et les filles. Les résultats de l'enquête font ainsi apparaître qu'une proportion plus importante de garçons que de filles affirme prendre régulièrement un petit-déjeuner $(54,6 \% v s 38,7 \%)$. Deux autres variables significativement orientées par le sexe des sujets ne semblent pas directement en rapport avec l'ensemble des autres effets observés (tous en lien avec les modes de sociabilité et les loisirs) : la fréquentation des lieux de culte et les choix (ou projets) d'orientation scolaire.

Si la fréquentation d'un lieu de culte est globalement importante dans l'ensemble de l'échantillon (78,9\%), elle est plus fréquemment déclarée chez les filles $(84,9 \%)$ que chez les garçons $(71,6 \%)$. A la question sur la filière d'étude visée, la non-réponse est plus fréquente chez les garçons (7,0\%) que chez les filles (1,3\%). Enfin, si la filière professionnelle attire autant les deux sexes, la filière générale semble privilégiée par les filles $(58,7 \% \mathrm{vs}$ $38,0 \%)$, alors que les garçons s'orientent plus fréquemment vers la filière technique $(10,5 \%$ vs $1,3 \%)$.

\begin{tabular}{|l|c|c|c|c|c|c|}
\hline & Non réponse & Générale & Technique & Professionnelle & Ne sais pas & Total \\
\hline Garçons & 7,0 & 38,0 & 10,5 & 28,4 & 16,2 & 100,0 \\
\hline Filles & 1,3 & 58,7 & 1,3 & 27,6 & 11,1 & 100,0 \\
\hline Total & 4,2 & 48,2 & 5,9 & 28,0 & 13,7 & 100,0 \\
\hline
\end{tabular}

Tri croisé : Sexe / Filière étude visée (tableau en pourcentage) Khi2 $=36,8$ ddl $=8$ p $=0,001$

Pour le reste, les écarts entre garçons et filles se situent essentiellement sur deux plans : celui des réseaux et des modes de sociabilités ; celui, plus spécifique, des pratiques physiques et sportives.

\subsection{Des loisirs et des modes de sociabilité marqués par le genre}

\subsubsection{Liens amicaux}

Les liens amicaux semblent se tisser dans des langues différentes pour les deux sexes. Les filles affirment plus fréquemment parler français avec les amis que les garçons (46,2\% d'entre elles contre 21,8\% d'entre eux), et les garçons plus fréquemment parler créole avec les amis que les filles $(73,8 \%$ d'entre eux pour 49,8\% d'entre elles).

\begin{tabular}{|c|c|c|c|c|c|}
\hline & Non rép. & Français & Créole & Anglais & Total \\
\hline Garçons & 3,5 & 21,8 & 73,8 & 0,9 & 100,0 \\
\hline Filles & 3,1 & 46,2 & 49,8 & 0,9 & 100,0 \\
\hline Total & 3,3 & 33,9 & 61,9 & 0,9 & 100,0 \\
\hline
\end{tabular}

Tri croisé : Sexe / Langue utilisée le plus souvent avec amis (tableau en pourcentage) Khi2=30,5 ddl $=10 \mathrm{p}=0,001$

Les réseaux amicaux ne sont également pas les mêmes. Pas tant par leur taille que par leur composition sexuée, puisque les garçons déclarent plus de « très bons amis garçons » que les filles, et les filles plus de « très bonnes amies filles » que les garçons. Ce résultat n'a rien de très surprenant. On constate cependant que le fait d'avoir de « très bons amis garçons » distingue davantage les deux sexes que le fait d'avoir « de très bonnes amies filles ». Est-ce à dire que les filles privilégient le fait de rester entre elles? 


\begin{tabular}{|c|c|c|c|c|c|c|c|}
\hline & Non rép. & 0 & $1-2$ & $3-5$ & $6-10$ & +10 & Total \\
\hline Garçons & 7,4 & 6,6 & 17,5 & 36,2 & 20,1 & 12,2 & 100,0 \\
\hline Filles & 3,1 & 13,8 & 37,8 & 27,6 & 12,0 & 5,8 & 100,0 \\
\hline Total & 5,3 & 10,1 & 27,5 & 31,9 & 16,1 & 9,0 & 100,0 \\
\hline
\end{tabular}

Tri croisé : Sexe / Nombre de très bon amis Garçons (tableau en pourcentage) Khi2 $=39,4$ ddl $=10$ p $=0,001$

\begin{tabular}{|c|c|c|c|c|c|c|c|}
\hline & Non rép. & 0 & $1-2$ & $3-5$ & $6-10$ & +10 & Total \\
\hline Garçons & 9,6 & 15,7 & 24,9 & 26,2 & 15,3 & 8,3 & 100,0 \\
\hline Filles & 2,2 & 3,1 & 25,3 & 36,9 & 23,6 & 8,9 & 100,0 \\
\hline Total & 5,9 & 9,5 & 25,1 & 31,5 & 19,4 & 8,6 & 100,0 \\
\hline
\end{tabular}

Tri croisé : Sexe / Nombre de très bonnes amies Filles (tableau en pourcentage) Khi2=37,6 ddl=10 p=0,001

\subsubsection{Lieux amicaux}

Pour les deux sexes, l'école est le lieu de provenance des amis le plus cité (95,69\% de moyenne pondérée), devant le quartier ou la section $(48,87 \%)$, le club de sport $(35,31 \%)$ et le groupe religieux $(27,38 \%)$. Mais les filles déclarent davantage avoir des amis qui proviennent de l'école $(97,7 \% \mathrm{vs} 93,7 \%)$, du groupe religieux $(31,4 \%$ vs $23,4 \%)$, des lieux de loisirs ( $13,2 \%$ vs $7,2 \%)$ et d'Internet (2,7\% vs $0,5 \%)$. Les amitiés des garçons semblent pour leur part plus fréquemment liées à la pratique dans un club sportif (49,1\% vs $21,4 \%)$.

\begin{tabular}{|l|c|c|c|c|c|c|c|c|c|}
\hline & Non rép. & Ecole & Section & Loisir & Club & Religion & Internet & Fête & Total \\
\hline Garçon & & 93,7 & 55,0 & 7,2 & 49,1 & 23,4 & 0,5 & 0,9 & 229,7 \\
\hline Fille & & 97,7 & 42,7 & 13,2 & 21,4 & 31,4 & 2,7 & 0,9 & 210,0 \\
\hline $\begin{array}{l}\text { Moyenne } \\
\text { pondérée }\end{array}$ & & 95,69 & 48,87 & 10,18 & 35,31 & 27,38 & 1,59 & 0,9 & \\
\hline
\end{tabular}

Tri croisé : Sexe / Lieux de provenance des amis Mult (tableau en pourcentage) Base Répondants. Khi2 $=70,2$ ddl $=20$ p=0,001

Globalement, les filles désignent plus fréquemment l'école $(35,9 \%$ vs $31,1 \%)$, la plage $(12,9 \%$ vs $8,9 \%)$, le lieu de culte $(8,3 \%$ vs $5,3 \%)$ et la maison $(2,2 \%$ vs $0,6 \%)$ comme lieu de rencontre des amis. Les garçons désignent de leur côté plus souvent le club $(13,5 \%$ vs $5,6 \%)$ et le terrain de sport $(9,8 \%$ vs 3,6\%).

\begin{tabular}{|l|c|c|c|c|c|c|}
\hline & Non rép. & Ecole & Terrain sp. & Club & Loisir & Lieu culte \\
\hline Garçons & 0,1 & 31,1 & 9,8 & 13,5 & 2,5 & 5,3 \\
\hline Filles & 0,2 & 35,9 & 3,6 & 5,6 & 4,2 & 8,3 \\
\hline Total & 0,2 & 33,3 & 6,9 & 9,8 & 3,3 & 6,7 \\
\hline
\end{tabular}




\section{S. FEREZ, S. RUFFIÉ ET M. LE HER}

\begin{tabular}{|l|c|c|c|c|c|c|}
\hline & Section & Plage & Place pub. & Internet & Maison & Total \\
\hline Garçons & 20,2 & 8,9 & 7,8 & 0,1 & 0,6 & 100,0 \\
\hline Filles & 17,3 & 12,9 & 9,5 & 0,5 & 2,2 & 100,0 \\
\hline Total & 18,9 & 10,8 & 8,6 & 0,3 & 1,3 & 100,0 \\
\hline
\end{tabular}

Tri croisé : Sexe / Lieux les plus fréquents de rencontre des amis (réponses multiples)

(tableau en pourcentage)

Khi2 $=61,2 \mathrm{ddl}=20 \mathrm{p}=0,001$

Le classement des lieux de rencontre des amis les plus fréquents confirme la plus grande importance que les filles accordent à l'école, à la plage et à la maison par rapport aux garçons. Les garçons soulignent au contraire la fréquence des rencontres dans la section ou le quartier $(17,9 \%$ d'entre eux classent ce dernier comme premier lieu de rencontre des amis, contre $4,4 \%$ des filles).

\begin{tabular}{|l|c|c|c|c|c|c|}
\hline & Non rép. & Ecole & Terrain sp. & club & loisir & lieu culte \\
\hline Garçons & 11,4 & 63,3 & 1,3 & 2,2 & & 1,7 \\
\hline Filles & 10,7 & 75,6 & & 0,4 & 0,4 & 1,8 \\
\hline Total & 11,0 & 69,4 & 0,7 & 1,3 & 0,2 & 1,8 \\
\hline
\end{tabular}

\begin{tabular}{|c|c|c|c|c|c|}
\hline & section & plage & Place pub. & Maison & Total \\
\hline Garçons & 17,9 & 0,4 & 1,7 & & 100,0 \\
\hline Filles & 4,4 & 4,4 & & 2,2 & 100,0 \\
\hline Total & 11,2 & 2,4 & 0,9 & 1,1 & 100,0 \\
\hline
\end{tabular}

Tri croisé : Sexe / Lieux les plus fréquents de rencontre des amis choix $n^{\circ} 1$ (tableau en pourcentage)

Khi2=37 ddl $=18 \mathrm{p}=0,005$

3.1.3. Activités partagées avec les amis

Concernant les activités réalisées avec les amis, si les dominos/cartes, la musique et les jeux sur ordinateur ou console sont plus mentionnés par les garçons que par les filles, c'est de loin la pratique du sport qui distingue le plus les premiers des secondes (72,6\% d'entre eux la mentionnent contre seulement 25,6\% d'entre elles). A l'inverse, les filles affirment plus fréquemment que les garçons faire les magasins (45,3\% vs 15,9\%), danser (40,8\% vs $11,5 \%)$ et dans une moindre mesure aller sur Internet avec leurs ami(e)s (66,8\% vs 49,1\%). L'ensemble de ces tendances est confirmé dans le classement par ordre de priorité des activités réalisées avec les amis. La discussion, Internet, la danse et les magasins apparaissent davantage dans les premiers choix d'activités chez les filles que dans les priorités des garçons, alors que, dans le même temps, le sport est confirmé comme un choix davantage mis en avant par les garçons.

\begin{tabular}{|l|c|c|c|c|c|c|}
\hline & Non rép. & Dominos / cartes & Danse & Discuter & TV & Sport \\
\hline Garçons & 17,5 & 8,3 & 1,7 & 34,9 & 2,2 & 22,3 \\
\hline Filles & 10,7 & 6,7 & 7,6 & 54,2 & 1,3 & 2,2 \\
\hline Total & 14,1 & 7,5 & 4,6 & 44,5 & 1,8 & 12,3 \\
\hline
\end{tabular}




\begin{tabular}{|c|c|c|c|c|c|c|c|}
\hline & Internet & Ciné & Musique & Magasins & Plage & Ordi / conso. & Total \\
\hline Garçons & 7,0 & 1,7 & 1,7 & 0,9 & & 1,7 & 100,0 \\
\hline Filles & 12,4 & & 0,4 & 4,0 & 0,4 & & 100,0 \\
\hline Total & 9,7 & 0,9 & 1,1 & 2,4 & 0,2 & 0,9 & 100,0 \\
\hline
\end{tabular}

Tri croisé : Sexe / Activités réalisées avec amis choix $n^{\circ} 1$ (tableau en pourcentage) Khi2=72,6 ddl $=24 \mathrm{p}=0,001$

\begin{tabular}{|l|c|c|c|c|c|c|c|}
\hline & Non rép. & Dominos / cartes & Danse & Discuter & TV & Sport & Internet \\
\hline Garçons & 27,9 & 6,1 & 1,7 & 15,7 & 5,2 & 21,0 & 14,0 \\
\hline Filles & 19,6 & 4,4 & 10,7 & 14,7 & 6,2 & 8,4 & 25,3 \\
\hline Total & 23,8 & 5,3 & 6,2 & 15,2 & 5,7 & 14,8 & 19,6 \\
\hline
\end{tabular}

\begin{tabular}{|c|c|c|c|c|c|c|}
\hline & Ciné & Musique & Magasins & Devoirs & Ordi / conso. & Total \\
\hline Garçons & 1,7 & 4,4 & 1,7 & & 0,4 & 100,0 \\
\hline Filles & 3,1 & 0,4 & 6,7 & 0,4 & & 100,0 \\
\hline Total & 2,4 & 2,4 & 4,2 & 0,2 & 0,2 & 100,0 \\
\hline
\end{tabular}

Tri croisé : Sexe / Activités réalisées avec amis choix n² (tableau en pourcentage) $\mathrm{Khi} 2=53 \mathrm{ddl}=26 \mathrm{p}=0,001$

\subsection{Des pratiques sportives différenciées pour les deux sexes}

\subsubsection{Une moindre pratique sportive chez les filles hors du temps scolaire}

Les garçons ont beaucoup plus fréquemment une pratique sportive hors EPS que les filles. Seuls 12,7\% d'entre eux n'en ont pas contre $41,3 \%$ des filles. Ils sont en outre surreprésentés parmi ceux qui déclarent un temps de pratique sportive supérieur à $4 \mathrm{~h}$ hebdomadaire (40,6\% d'entre eux, pour $12,5 \%$ des filles).

\begin{tabular}{|l|c|c|c|c|c|c|c|c|}
\hline & Non rép. & $0-2 \mathrm{~h}$ & $2 \mathrm{~h}$ & $2-4 \mathrm{~h}$ & $4-6 \mathrm{~h}$ & $6-8 \mathrm{~h}$ & $+8 \mathrm{~h}$ & Total \\
\hline Garçons & 14,8 & 9,2 & 19,2 & 16,2 & 17,9 & 12,2 & 10,5 & 100,0 \\
\hline Filles & 41,3 & 11,6 & 20,4 & 14,2 & 7,6 & 2,2 & 2,7 & 100,0 \\
\hline Total & 28,0 & 10,4 & 19,8 & 15,2 & 12,8 & 7,3 & 6,6 & 100,0 \\
\hline
\end{tabular}

Tri croisé : Sexe / Temps APS hebdo hors EPS (tableau en pourcentage) Khi2 $=65,1 \mathrm{ddl}=12 \mathrm{p}=0,001$

\subsubsection{Des goûts sportifs sexués}

Des différences significatives entre les sexes apparaissent par ailleurs du point de vue des goûts sportifs. Les garçons affichent une plus grande sympathie pour les sports de contact $(18,9 \%)$ et un moindre intérêt pour les sports sans contact $(6,1 \%)$. Les filles affirment pour leur part davantage leur préférence pour les activités sans contact (14,3\%) et paraissent moins enclines à choisir les pratiques de contact (10,5\%). Dans le même temps, alors que les premiers privilégient plus la dimension de la « technique » que les secondes $(14,4 \%$ d'entre eux contre $9,1 \%$ d'entre elles), celles-ci sont plus attachées à la dimension physique de l'activité (15,8\% d'entre elles contre $10,5 \%$ d'entre eux). Une question demeure cependant quant au sens que les filles attribuent à cette dimension « physique » : cette dernière est-elle associée à des aspects fonctionnels de force et de vitalité ou à des considérations esthétiques? 


\section{S. FEREZ, S. RUFFIÉ ET M. LE HER RECHERCHES}

3.2.3. Des lieux de pratique sportive différenciés selon le sexe

Lorsqu'il y a une pratique sportive hors du temps scolaire, les filles sont beaucoup plus nombreuses que les garçons à ne pas en préciser le lieu. Alors qu'elles sont autant que les garçons à pratiquer à la plage et à la maison, elles pratiquent moins fréquemment dans la rue, dans la nature, sur un terrain de sport ou en club.

\begin{tabular}{|l|c|c|c|c|c|c|c|c|c|}
\hline & Non rép. & Plage & Nature & Rue & Terrain & Maison & Club & Salle & Total \\
\hline Garçons & 15,07 & 26,1 & 26,6 & 25,6 & 41,2 & 44,2 & 58,8 & 1,0 & 223,6 \\
\hline Filles & 73,07 & 31,5 & 17,7 & 14,6 & 23,1 & 53,1 & 43,1 & 1,0 & 184,6 \\
\hline
\end{tabular}

Tri croisé : Sexe / Lieux d'activités hors EPS (réponses multiples)

(tableau en pourcentage)

Base Répondants. Khi2 $=87,8 \mathrm{ddl}=14 \mathrm{p}=0,001$

Les garçons sont plus nombreux à déclarer pratiquer en premier lieu en club $(34,1 \%$ d'entre eux contre $19,6 \%$ des filles) et dans la nature ( $8,3 \%$ d'entre eux contre $1,3 \%$ des filles). Leur second lieu de pratique est plus souvent le terrain de sport $(15,7 \%)$ et la rue (10\%) que les filles (respectivement $4,9 \%$ et $3,1 \%$ d'entre elles).

3.2.4. Une pratique entre personnes de même sexe (ou non mixte)

En ce qui concerne la pratique sportive hors EPS, les garçons déclarent plus souvent pratiquer avec des amis garçons (31,3\% d'entre eux contre $9,7 \%$ des filles) et des partenaires de club $(28,1 \%$ d'entre eux contre $15,4 \%$ des filles) et les filles davantage pratiquer avec des amies filles (16,3\% d'entre elles contre 4,9\% des garçons) ou avec leur mère $(7,7 \%$ d'entre elles contre 3,0\% des garçons). Cette différence concerne essentiellement les partenaires les plus fréquents.

\begin{tabular}{|l|c|c|c|c|c|c|}
\hline & Non rép. & Amis Garçons & Parten. club & Seul & Père & Mère \\
\hline Garçons & 27,1 & 21,8 & 33,2 & 10,0 & 3,5 & 1,3 \\
\hline Filles & 51,6 & 2,2 & 17,3 & 12,0 & 2,2 & 4,4 \\
\hline Total & 39,2 & 12,1 & 25,3 & 11,0 & 2,9 & 2,9 \\
\hline
\end{tabular}

\begin{tabular}{|c|c|c|c|c|}
\hline & Amis Filles & Parten. famille & Etranger & Total \\
\hline Garçons & 0,4 & 1,3 & 1,3 & 100,0 \\
\hline Filles & 9,3 & 0,9 & & 100,0 \\
\hline Total & 4,8 & 1,1 & 0,7 & 100,0 \\
\hline
\end{tabular}

Tri croisé : Sexe / Partenaire de pratique hors EPS choix $n^{\circ} 1$ (tableau en pourcentage) Khi2 $=89,6$ ddl $=16$ p $=0,001$

\begin{tabular}{|c|c|c|c|c|c|c|}
\hline & Non rép. & Amis Garçon & Parten. club & Seul & Père & Mère \\
\hline Garçons & 59,4 & 19,2 & 7,0 & 5,7 & 3,9 & 2,2 \\
\hline Filles & 74,2 & 5,3 & 1,3 & 4,0 & 2,7 & 3,6 \\
\hline Total & 66,7 & 12,3 & 4,2 & 4,8 & 3,3 & 2,9 \\
\hline
\end{tabular}




\begin{tabular}{|c|c|c|c|c|}
\hline & Amis Filles & Parten. famille & Etranger & Total \\
\hline Garçons & 2,2 & 0,4 & & 100,0 \\
\hline Filles & 7,6 & 1,3 & & 100,0 \\
\hline Total & 4,8 & 0,9 & & 100,0 \\
\hline
\end{tabular}

Tri croisé : Sexe / Partenaire de pratique hors EPS choix n 2 (tableau en pourcentage) Khi2 $=39,1$ ddl $=16$ p $=0,001$

\section{DISCUSSION}

Les résultats révèlent qu'il n'existe, dans l'échantillon étudié, quasiment pas de différences entre les deux sexes au niveau des habitudes alimentaires et sur le plan des pratiques sanitaires stricto sensu. La logique du genre ne semble ainsi ici ne concerner que des aspects liés à la sociabilité, et plus spécifiquement à la construction de cette dernière autour d'usages culturels et de loisirs différenciés selon le sexe. Pour autant, cela ne signifie pas qu'elle n'a pas d'effets sanitaires, mais que ces derniers ne sont pas directs : ils passent par l'élaboration d'un rapport sexué aux pratiques de loisirs, et notamment à l'activité physique, en dehors de toute motivation sanitaire pour les sujets.

\subsection{Construction sociale du genre et effets sanitaires}

La seule différence significative observée entre les sexes sur des aspects alimentaires ou sanitaires concerne le fait que les garçons déclarent plus fréquemment prendre régulièrement un petit déjeuner. Le sexe a un effet sur deux autres variables apparemment sans lien avec la sociabilité de genre et la pratique de loisirs : la fréquentation des lieux de culte et les projets d'orientation scolaire. A l'issue de l'analyse, il apparait toutefois que ces deux variables orientent fortement les logiques de genre qui organisent les sociabilités et l'investissement dans les pratiques de loisirs. Ainsi, la fréquentation plus importante des lieux de culte chez les filles ne marque pas une différence avec les garçons au niveau des croyances religieuses. Elle correspond par contre à des différences de sociabilités. Les filles déclarent ainsi davantage d'ami(e)s provenant du lieu de culte, lieu où elles rencontrent par ailleurs plus fréquemment leurs ami(e)s (qui sont plus souvent des filles).

De même, les écarts dans les projets d'orientation scolaire débouchent sur une ségrégation (relative) des groupes de sexe - qui sont conduits dans des filières et donc des classes différentes - et des interactions sexuées à partir du lycée. La prévalence donnée par les filles à la filière générale, alors que les garçons privilégient la filière technique, atteste parallèlement d'une opposition entre l'abstrait et le concret (le « théorique » et le « pratique ») qui structure et que structurent aussi les goûts en matière de loisirs (Blöss, 2002). Le féminin s'élabore dans l'association au général, à l'abstraction et au religieux, quand le masculin s'élabore dans le rapport au pragmatique, à la technique et au sport.

Si cette construction dichotomique du genre n'a pas permis, dans cette étude (en fonction des catégories d'enquête utilisées), d'établir de lien avec des habitudes alimentaires et des pratiques sanitaires, elle produit cependant des effets qui, bien que résultant d'une logique qui leur est propre (celle de la signification sexuée des pratiques) peuvent avoir des effets sur la santé. Au final, si la socialisation de genre a peu d'impact direct sur les aspects alimentaires et sanitaires immédiats - l'origine sociale et l'appartenance socioprofessionnelle semblent ici plus décisifs (Bourdieu, 1979 ; Boltansky, 1971 ; Perrin et al., 2002) -, cela ne signifie pas que les logiques qu'elle produit, notamment en termes de rapport à l'activité physique, n'ont pas d'effets plus indirects et différés sur la santé (et en particulier sur l'obésité et le surpoids).

\subsection{L'inscription de l'activité physique dans les sociabilités de genre}

La quasi-totalité des variables impliquées dans les tris croisés significatifs avec la variable « sexe » concerne deux indicateurs : le niveau d'activité physique ou de sédentarité ; les réseaux amicaux, de pratiques de loisirs et les usages culturels. Concernant le premier, il apparaît que les filles déclarent moins souvent une pratique sportive hors du temps scolaire et des durées de pratique hebdomadaire moins importantes que les garçons. A ces écarts au niveau de l'engagement sportif, correspondent des différences de goûts : les garçons se montrent plus attachés au contact et à la technique, les filles à la dimension physique de l'activité. Il n'est cependant pas possible de savoir la signification exacte que les filles confèrent à cette dernière dimension : le "physique » renvoie-t-il ici à la dépense énergétique et à la vitalité, ou bien correspond-t-il à une préoccupation esthétique ?

Moins fréquente, et selon des goûts spécifiques, la pratique physique des filles se fait par ailleurs dans des lieux différents : plus souvent à la plage et à la maison, alors que les garçons s'investissent plus dans la rue, dans la nature, sur un terrain de sport ou en club. Le choix des espaces apparaît surdéterminé par le genre (Vernier, 1998). 


\section{S. FEREZ, S. RUFFIÉ ET M. LE HER}

Aux filles des lieux qui suggèrent un caractère privatif et aux garçons des espaces publics. La plage pourrait donner lieu à controverse, étant un espace par définition public. Cependant le caractère très familial des sorties à la plage le week-end en Guadeloupe peut laisser entrevoir un aspect privatif de la plage, ou du moins familial, qui s'accorde avec les orientations de genre. Enfin, un écart apparaît concernant les partenaires de la pratique hors E.P.S. : les amis de même sexe sont, pour les garçons comme pour les filles, les partenaires privilégiés (tandis que les filles affirment plus souvent que les garçons pratiquer avec leur mère).

$\mathrm{Au}$ total, la pratique physique et sportive s'inscrit clairement dans des sociabilités de genre qui génèrent la ségrégation des groupes de sexes. Les réseaux amicaux apparaissent ainsi comme fortement sexués. Les « très bons amis » sont essentiellement du même sexe, même si les garçons semblent plus volontiers déclarer s'insérer dans des réseaux amicaux féminins que l'inverse. Ce plus grand nombre d'amies de l'autre sexe déclaré par les garçons interroge. Est-ce que la notion de "très bons amis" a la même signification chez les garçons et les chez filles ou est-ce que la moindre présence d'amitiés masculines chez les filles indique des statuts de genre dissymétriques qui orientent la signification dans les rapports de sexe ? Les garçons ne doivent-ils pas, au regard de leur identité de genre, déclarer davantage d'amies filles en vue de renforcer une forme de rapports de supériorité et de contrôle du genre féminin ? (Bourdieu, 1998)

Des écarts entre les sexes se manifestent en outre au sujet des lieux de provenance et de rencontre des amis : l'école, la plage, la maison, le groupe religieux et le lieu de culte sont plus cités par les filles, le terrain de sport et la pratique en club sportif par les garçons. Mais ce sont également les activités partagées avec ses amis qui différent. Du côté des garçons, un privilège est donné aux dominos/cartes, à la musique, aux jeux sur ordinateur ou sur console vidéo, et surtout au sport. Les filles affirment pour leur part davantage discuter, danser, faire les magasins et aller sur Internet avec leurs ami(e)s.

On le voit, la pratique sportive s'inscrit dans des modes de sociabilités, d'interactions amicales et des usages des loisirs fortement sexués. Il est intéressant de noter que les filles, qui donnent plus d'importance que les garçons au fait de discuter entre ami(e)s (alors que ces derniers privilégient plus le fait d'avoir une activité sportive avec leurs copains), déclarent plus fréquemment utiliser la langue française dans les rapports amicaux que les garçons, pour qui le créole est plus souvent mentionné. Apparaît ainsi la manière dont la socialisation de genre interfère sur les liens qui se tissent entre sport et identité culturelle (Dumont, 2002). Apparaît aussi combien l'engagement ou l'absence d'investissement dans l'activité physique et sportive prend sens au regard de logiques sociales où interfèrent des aspects socioculturels (liés à une identité locale) et des aspects socio-sexués (liés à l'identité de genre).

\section{CONCLUSION}

L'enquête réalisée auprès d'une population de jeunes Guadeloupéens scolarisés montre combien le sport participe d'une construction traditionnelle des identités de genre chez ce public. Outre sur l'engagement sportif à proprement parler, cette construction a des effets importants sur les modes de sociabilité et les rapports aux pratiques culturelles et de loisir. Elle ne semble par contre pas peser directement sur les habitudes alimentaires et les pratiques sanitaires. Mieux saisir les effets sur la santé de ces « styles de vie » différenciés selon le genre, en particulier dans le domaine de l'activité physique et sportive, aide sans doute à comprendre les difficultés qui peuvent exister à les faire évoluer. La pratique sportive ne peut ici s'appréhender comme un simple moyen technologique, l'outil absolument neutre d'une politique sanitaire, car elle s'inscrit a priori dans des systèmes de signification sociale. Comprise comme un vecteur des goûts culturels, elle offre une bonne image de la structuration de la société (Veenstra, 2007).

Les discours de prévention sanitaire et d'incitation à la pratique physique se heurtent ainsi à des logiques qui leur échappent : celles produites par l'arrangement des sexes (Goffman, 2002) et par la construction d'habitus sexué (Muel-Dreyfus, 2003). Dans la population étudiée, l'investissement sportif n'est pas massivement inscrit par une perspective hygiénique et sanitaire, ni orienté par elle. L'espace sportif offre avant tout un lieu d'incorporation de la structure sociale de genre (Brown, 2006). Aussi, comme l'observent Daniels et Leaper (2006), l'acceptation dans le groupe de pairs explique en grande partie les liens qui existent entre participation sportive et élévation de l'estime de soi globale. Or, ce rôle des pairs n'incite pas à la transformation des attitudes sociales concernant la participation sportive en fonction du genre. Au travers de l'activité physique et sportive apparaît finalement la manière dont les réseaux amicaux et la socialisation secondaire (à la fois dans et en marge de l'école) prennent le relais de la socialisation primaire du genre exercée au sein de la famille. 
- Ara, I., et al.(2007). Adiposity, physical activity, and physical fitness among children from Aragón, Spain. Obesity, 15(8), 1918-24.

- Atkinson, M. (2007). Playing with fire: Masculinity, health, and sports supplements. Sociology of Sport Journal, 24(2), 165-186.

- Ball, K., Crawford, D. et Kenardy, J. (2004). Longitudinal relationships among overweight, life satisfaction, and aspiration in young women. Obesity research, 12(6).

- Beech, B.M., et al. (2004). Parental Cultural Perspectives in Relation to Weight-Related Behaviors and Concerns of African-American Girls. Obesity research, 12, 7-19.

- Blöss, T. (2002). La Dialectique des rapports hommes-femmes (éd.). Paris : PUF, coll. Sociologie d'aujourd'hui.

- Boltanski, L. (1971). Les Usages sociaux du Corps. Annales ESC, 26, 205-233.

- Bonaccorsi, G., et al. (2007) Body composition, nutritional habits and functional abilities in a sample of eight year old children [translated from Italian]. Ann Ig, 19(3), 215-23.

- Bourdieu, P. (1998). La Domination masculine. Paris : Seuil.

- Brown, D. (2006). Pierre Bourdieu's "masculine domination" thesis and the gendered body in sport and physical culture. Sociology of Sport Journal, 23(2), 162-188.

- Clark, S., Paechter, C. (2007). 'Why can't girls play football?' Gender dynamics and the playground. Sport, Education and Society, 12(3), 261-276.

- Clayton, B., Humberstone, B. (2007). 'Gender and race - What's that to do with football studies?' Contested 'knowledges' in sport and leisure curricula in HE. Gender and Education, 19(4), 513-533.

- Coakley, J. (2006). The good father: Parental expectations and youth sports. Leisure studies, 25(2), 153-163.

- Daniels, E., Leaper, C. (2006). A longitudinal investigation of sport participation, peer acceptance, and selfesteem among adolescent girls and boys. Sex roles, 55(11-12), 875-880.

- Dowda, M., Ainsworth, B.E., Addy, C.L. (2001). Environmental Influences, Physical Activity, and Weight Status in 8- to 16 year olds. Arch pediatr Adolesc med, 155, 711-717.

- Dumont, J. (2002). La Diffusion du sport en Guadeloupe: Enjeux culturels autour de la départementalisation, Paris, L'Harmattan, Coll. Espaces et temps du sport.

- Ferez, S. (2004). De l'expression corporelle aux Activités Physiques Artistiques : Subversion sexuée et différenciation sexuée en Education Physique et Sportive. STAPS, 66, 113-128.

- Ferez, S. (2007). Le Corps homosexuel en-jeu. Sociologie du sport gay et lesbien (Préface d'Eric Fassin). Nancy : PUN, coll. Epistémologie du corps.

- Ferez, S. (2008). Le masculin. In B. Andrieu \& G. Boetsch (dir.) Le Dictionnaire du corps (pp. , 205-207). Paris : CNRS Editions (réédition augmentée).

- Forbes, G.B., Adams-Curtis, L.E., Pakalka, A.H., White, K.B. (2006). Dating agression, sexual coercion, and agression-supporting attitudes among college men as a function of participation in agressive high school sports. Violence Against Woman, 12(5), 441-455.

- Giraud, M. (1999). Une construction coloniale de la sexualité. Actes de la recherhe en sciences sociales, 128(1), 46-55.

- Goffman, E. (2002). L'Arrangement des sexes (1 $1^{\text {ere }}$ édition en 1977). Paris : La Dispute, coll. Le genre du monde.

- Gordon-Larsen, P. (2001). Obesity-Related Knowledge, Attitudes, and Behaviors in Obese and Non-obese Urban Philadelphia Female Adolescents, Obesity Research, 9(2).

- Grindstaff, L., West, E. (2006). Cheerleading and the gendered politics of sport. Social Problems, 53(4), 500-518.

- Hanson, S.L. (2007). Young women, sports, and science. Theory into Practice, 46(2), 155-161.

- Hoeber, L. (2007). Exploring the gaps between meanings and practices of gender equity in a sport organization. Gender, Work and Organization, 14 (3), 259-280.

- Kosti, R.I., et al. (2007). Dietary habits, physical activity and prevalence of overweight/obesity among adolescents in Greece: The Vyronas study. Med Sci Monit, 13(10), 437-444.

- Larson, H. (2006). A history of the present on the "Sportsman" and the "Sportswoman". Historical Social Research, 31(1), 209-229.

- Liston, K. (2006). Sport and gender relations. Sport in Society, 9(4), 616-633.

- Muel-Dreyfus, F. (2003). Une écoute sociologique de la psychanalyse. In P. Encrevé et R.-M. Lagrave, Travailler avec Bourdieu (pp. 227-235.). Paris : Flammarion.

- Müller, M. (2006). Geschlecht als Leistungsklasse: Der Kleine Unterschied und Sein Großen Folgen am Beispiel der "gender Verifications" im Leistungssport [Gender as class in competitive sports: Gender verifications in sports as an example of the "small difference and its big consequences"]. Zeitschrift fur Soziologie, 35(5), 392-412. 


\section{S. FEREZ, S. RUFFIÉ ET M. LE HER}

- Mulot, S. (2000). "Je suis la mère, je suis le père" : l'énigme matrifocale. Relations familiales et rapports de sexes en Guadeloupe, Thèse de doctorat en anthropologie sociale et en ethnologie, Paris, EHESS.

- Padgett, J., Biro, F.M. (2003). Different Shapes in Different Cultures: Body Dissatisfaction, Overweight, and Obesity in African-America and Caucasian Females. J Pediatr Adolesc Gynecol, 16, 349-354.

- Paeratakul, S., et al. (2002). Sex, Race/Ethnicity, Socioeconomic Status, and BMI in Relation to Self-Perception of Overweight. Obesity Research, 10(5), 345-350.

- Perrin, C, et al. (2002). Lifestyle patterns concerning sports and physical activity, and perceptions of health. Social and Preventive Medicine, 47, 162-171.

- Perry, A.C., Rosenblatt, E.B., Wang, X. (2004). Physical, Behavioral, and Body Image Characteristics in a TriRacial Group of Adolescent Girls. Obesity research, 12(10), 1670-1679.

- Pourette, D. (2002). Hommes et femmes de la Guadeloupe en Ile-de-France. Pratiques liées au corps, relations entre les sexes et attitudes face au risque de contamination par le VIH, Thèse de doctorat en ethnologie et anthropologie sociale. Paris : EHESS.

- Pourette, D. (2004). Les notions de nature et de contre-nature dans la pensée guadeloupéenne. Rôles de genre, sexualité et sida. Les Cahiers des Anneaux de la Mémoire, 7, 143-171.

- Robertson, S. (2003). 'If I let a goal in, I'll get beat up': Contradictions in masculinity, sport and health. Health Education Research, 18 (6), 706-716.

- Ruffié, S., et al (soumis, mai 2008). L'usage du sport comme revendication à l'intégration active. Sport et « handicap » en Guadeloupe. Sciences Sociales et Santé.

- Sanchez-Johnsen, L.A.P., et al. (2004). Ethnic Differences in Correlates of Obesity between Latin-American and Black Women. Obesity Research, 12(4), 652-660.

- Schmalz, D.L., Kerstetter, D.L. (2006). Girlie girls and manly men: Children's stigma consciousness of gender in sports and physical activities. Journal of Leisure Research, 38(4), 536-557.

- Simeon, D.T., et al. (2003). Body image of adolescents in a multi-ethnic Caribbean population. European journal of clinical nutrition, 57, 157-162.

- Strauss, R.S. (2000). Childhood Obesity and Self-Esteem. Pediatrics, 105(1).

- Strauss R.S., Pollack H.A. (2003) Social marginalization of overweight children. Arch pediatr adolesc med, $157,746-752$.

- Taylor, R.W., et al. (2006). Reducing weight gain in children through enhancing physical activity and nutrition: the APPLE project. Int J Pediatr Obes, 1(3), 146-152.

- Terret, T. (2005). Sport et genre, vol.1, « La Conquête d'une citadelle masculine » (dir.), Paris : L'Harmattan, coll. Espace et temps du sport.

- Veenstra, G. (2007). Who the heck is don bradman? Sport culture and social class in British Columbia, Canada. Canadian Review of Sociology and Anthropology, 44 (3), 319-343.

- Vernier, B. (1998). Représentation mythique du monde et domination masculine chez les Pomaques grecs. Actes de la Recherche en sciences sociales, 125, 74-98.

- Webb, L., Macdonald, D. (2007). Dualing with gender: Teacher' work, careers and leadership in physical education. Gender and Education, 19 (4), 491-512.

- Williams, J. (2006). An equality too far ? Historical and contemporary perspectives of gender inequality in British and international football. Historical Social Research, 31(1), 151-169. 